\title{
A Study on the Static Stability of Scissor Lift
}

\author{
Wei Zhang ${ }^{*}, 1,2,3$, Chen Zhang ${ }^{2}$, Jiangbo Zhao ${ }^{3}$ and Chunzhi $\mathrm{Du}^{1}$ \\ ${ }^{1}$ Airport College, Civil Aviation University of China, 100 Road Xunhai, Tianjin, P.R. China \\ ${ }^{2}$ Sino-European Institute of Aviation Engineering, 100 Road Xunhai, Tianjin, P.R. China \\ ${ }^{3}$ Aviation Ground Special Equipment Research 100 Road Xunhai, Tianjin, P.R. China
}

\begin{abstract}
This research paper studies the static stability of six kinds of scissor lifts with one input force of hydraulic actuator by having the input on the lines of the nodes of the scissor lifts. Firstly, the static stability of single scissor arm is studied by using energy method and modeling method in the software Nastran. The stability results of two methods are then compared. Subsequently, the scissor lift models with hydraulic actuators are prepared to analyze the static stability. The static stability of six kinds of scissor lifts is compared. It is thus found that the results of the overall model are closer to the actual situation. The analysis of single arm models has made it easier to compare the theoretical solutions and modeling solutions thus the study on stability of single scissor arm has been proved to be meaningful.
\end{abstract}

Keywords: Energy method, Finite element method, Scissor lift, Static stability.

\section{INTRODUCTION OF SCISSOR LIFTS WITH DIFFERENT INPUT VECTORS}

Scissor lifting mechanism is a typical lifting machine [16] which has many advantages such as stable structure [4], reliable operation [1], high efficiency [2, 3, 5] and low failure rate $[2,6]$, etc. As a key component of platforms, the stability of scissors lifting mechanism determines the safety of the platform equipment $[1,6]$. The instablility of scissor lift can cause physical harm to the agency staff and can even cause death. In this paper, models of single scissor arm are prepared by using Finite Element software. The critical load is calculated by conducting buckling analysis. The theoretical results of critical loads are calculated by using energy method. The two results of modeling and energy methods are compared. The FEM models of the total scissor lifts mechanism are prepared and the stability of scissor lifts with different input vectors is compared.

There are six different kinds of scissor lifts in total having one input force of hydraulic actuator and the input is on the lines of the nodes of the scissor lifts as shown in Fig. (1).

\section{RELATED DATA OF MODELS}

The main parts of scissor lifts are two scissor arms, a hydraulic actuator and a platform. The models are simplified by two dimension models with a hypothesis that the load $\mathrm{W}$ is fixed in the middle of the platform. The actuator force is $\mathrm{P}$ and the length of two scissor arms are $2 L$ with their numbers as shown in Fig. (2).

*Address correspondence to this author at the Airport College, Civil Aviation University of China, 100 Road Xunhai, Tianjin, P.R. China; Tel: +86 22 24092477; Fax: +86 22 24092886;

E-mail: caucaslm@163.com
The first kind of scissor lift is shown with the angle between ground and the scissor arm and the blue line represents the hydraulic actuator. All the references of the scissor lift are shown in Fig. (2).

The material of the scissor arm is aluminum alloy, with Young's modulus $70 \mathrm{GPa}$, Poisson ratio 0.3 and density $2.5 \times 10^{-6} \mathrm{~kg} / \mathrm{mm}^{3}$. The material of the hydraulic actuator is steel with Young's modulus $200 \mathrm{GPa}$, Poisson ratio 0.3, and density $7.8 \times 10^{-6} \mathrm{~kg} / \mathrm{mm}^{3}$.

Based on the equation of input hydraulic force and the load, following can be obtained

$$
P=\frac{2 W l}{N \sin \theta+M \cos \theta \tan \alpha}
$$

The references $M$ and $N$ in the six cases are shown in Table 1.

For all the rest of the research, the data of all references are given: $l=600 \mathrm{~mm}, W=2000 \mathrm{~N}$, the value varying from 15 to 45 degrees. All the other data of references are shown in Table 2.

The cross section of the scissor arm is shown in Fig. (3), with data $B=30 \mathrm{~mm}, H=50 \mathrm{~mm}, b=24 \mathrm{~mm}, h=44 \mathrm{~mm}$.

The area of the cross section and the inertial moment are given as:

$$
\begin{aligned}
& A=H B-h b=444 \mathrm{~mm}^{2} \\
& I=\frac{B H^{3}-b h^{3}}{12}=142000 \mathrm{~mm}^{4}
\end{aligned}
$$

\section{STATIC ANALYSIS OF RIGID BODY}

In order to analyze the stability of scissor arms, the internal forces of the nodes must be calculated. Each of the 

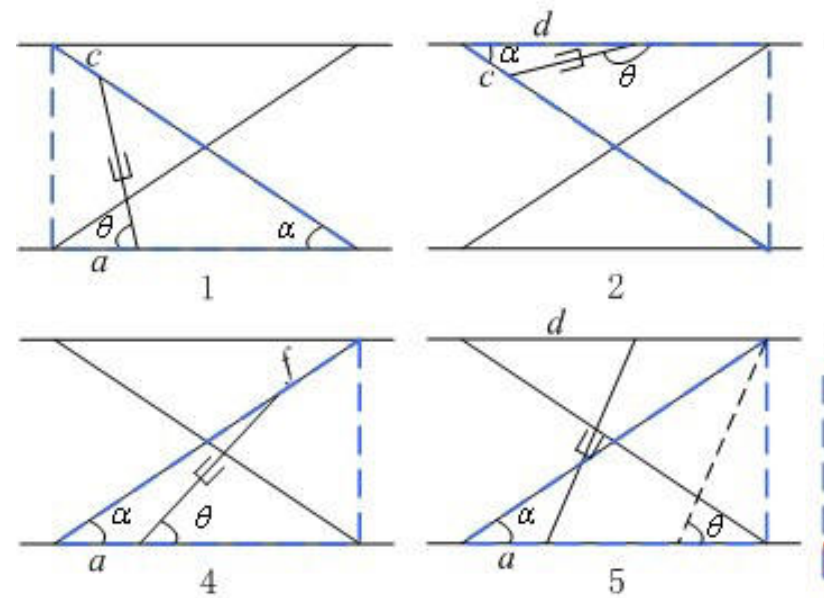

2

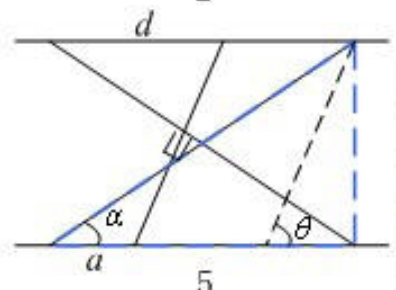

5

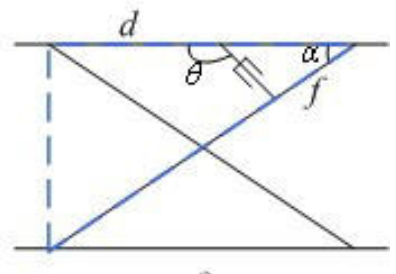

3

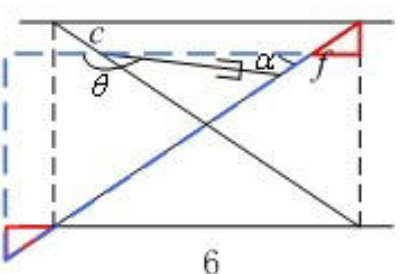

Fig. (1). Characteristic triangles of 6 different input configurations.

five nodes is given a number. In the coordinate system, the forces can be decomposed to sand. Since $W$ is in the middle of the platform, the hypothesis is given. The first case is shown in Fig. (4).

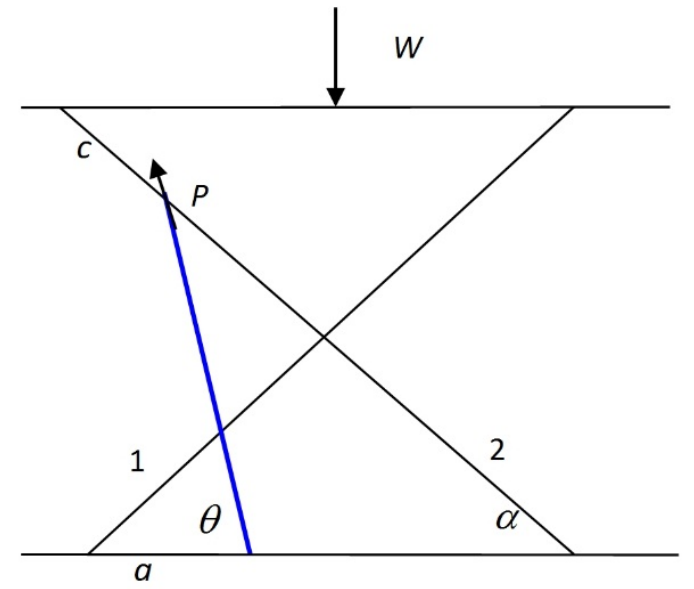

Fig. (2). Characteristic triangles of 6 different input configurations.

Table 1. Expressions of references between hydraulic force and load.

\begin{tabular}{|c|c|c|c|c|c|c|}
\hline Case & $\mathbf{1}$ & $\mathbf{2}$ & $\mathbf{3}$ & $\mathbf{4}$ & $\mathbf{5}$ & $\mathbf{6}$ \\
\hline \hline$M$ & $-c$ & $c$ & $f-2 l$ & $2 l-f$ & 0 & $c+f-2 l$ \\
\hline$N$ & $2 l-c$ & $c$ & $f$ & $2 l-f$ & $2 l$ & $f-c$ \\
\hline
\end{tabular}

Table 2. Expressions of references of the six scissors.

\begin{tabular}{|c|c|c|c|c|c|c|}
\hline Reference & $\mathbf{1}$ & $\mathbf{2}$ & $\mathbf{3}$ & $\mathbf{4}$ & $\mathbf{5}$ & $\mathbf{6}$ \\
\hline \hline$c(\mathrm{~mm})$ & 200 & 200 & & & & 200 \\
$f(\mathrm{~mm})$ & & & 200 & 200 & & 400 \\
$a(\mathrm{~mm})$ & 300 & & & 548 & 200 & \\
$d(\mathrm{~mm})$ & & 300 & 548 & & 300 & \\
\hline
\end{tabular}

$F y_{1}=F y_{2}=-\frac{W}{2}$

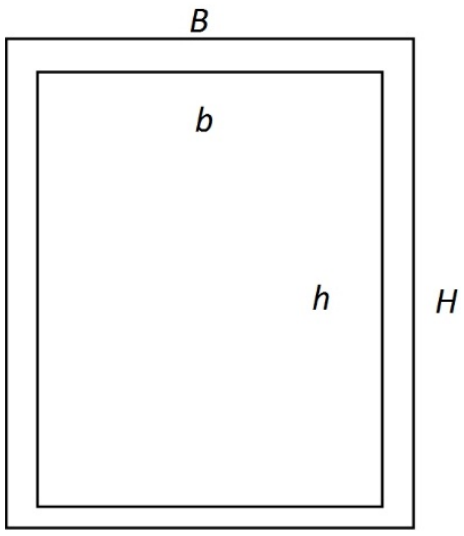

Fig. (3). Characteristic triangles of 6 different input configurations.

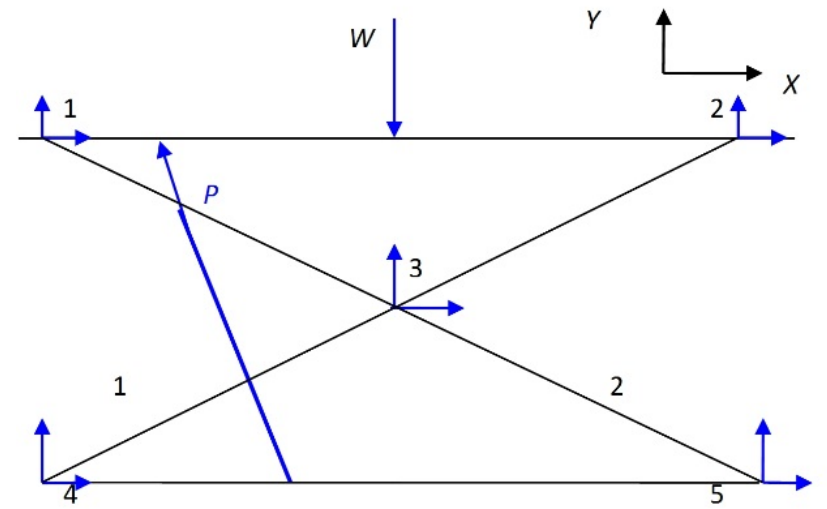

Fig. (4). Coordinate system and number of nodes in the scissor lifts.

The equilibrium of the force for the whole scissor:

$-P \cos \theta+F x_{4}=0$

$-W+P \sin \theta+F y_{4}+F y_{5}=0$

The equilibrium of the moment for the whole scissor is calculated as:

$-W \frac{L \cos \theta}{2}+F y 5 L \cos \alpha+P a \sin \theta=0$

The equilibrium of the force for the scissor arm number 1 : 
$F x_{3}=-F x_{4}$

$F y_{3}+F y_{4}-\frac{W}{2}=0$ 2:

The equilibrium of the force for the scissor arm number

$-\frac{W}{2}+F y_{5}-F y_{3}+P \sin \theta=0$

From (2) to (7), the internal forces of nodes represented by $W$ and $P$ can be calculated (See Table 3 ).

Table 3. Expressions of internal forces for the first case.

\begin{tabular}{|c|c|c|}
\hline Internal Forces of Nodes & $F x$ & $F y$ \\
\hline \hline 1 & 0 & $-\frac{W}{2}$ \\
\hline 2 & 0 & $-\frac{W}{2}$ \\
\hline 3 & $P \sin \theta$ & $P \sin \theta-P \frac{a \sin \theta}{L \cos \theta}$ \\
\hline 4 & $P \sin \theta$ & $\frac{W}{2}-P \sin \theta+P \frac{a \sin \theta}{L \cos \theta}$ \\
\hline 5 & 0 & $\frac{W}{2}-P \sin \theta$ \\
\hline
\end{tabular}

\section{STABILITY ANALYSIS OF SCISSOR ARM BY ENERGY METHOD}

The total potential energy of an object is equal to the puissance of all forces from the stress state position to unstressed position. Since both the external force and internal force contribute to the puissance, the total potential energy expression is:

$\Pi=U+V$

where $\mathrm{U}$ is internal potential energy and $\mathrm{V}$ is outer energy. For an elastic body, $U$ equals to outer puissance, therefore $\Pi=-U$

For a scissor arm, when the derivative of potential energy to the axial force equals 0 , the arm is in equilibrium. When the second derivative of potential energy to the axial force is bigger than 0 , the arm is in stable equilibrium. In this case, the axial load is the critical load.

The first derivative of internal energy is:

$d U=\frac{F^{2} d x}{2 E A}+\frac{M^{2} d x}{2 E I}$

where $F$ is the axial force, $M$ is the moment of the arm.

Suppose the scissor arm can be divided into two parts of length $l$, the integration of internal energy is:

$U=\int_{0}^{l}\left(\frac{F^{2} d x}{2 E A}+\frac{M_{1}^{2} d x}{2 E I}\right)+\int_{l}^{2 l}\left(\frac{F^{2} d x}{2 E A}+\frac{M_{2}^{2} d x}{2 E I}\right)$ where $M_{1}$ and $M_{2}$ are moments on the two parts of the arm.

The critical force $F$ can be calculated by the derivation of $U$ to $F$. When the derivation equals 0 , the relevant force equals the critical load.

The first case is taken as an example. In order to analyze the stability of scissor arm, simplification of boundary condition is given in Fig. (5).

By analyzing the scissor arm number 1

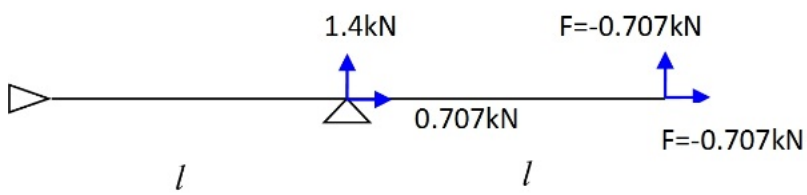

Fig. (5). Boundary condition of the scissor arm number 1 of first case.

By defining $\mathrm{V}$ as the deflection of the scissor arm, the following can be obtained:

$$
\begin{aligned}
& M_{2}=F\left(V_{\max }+V_{2}+l-x\right) \\
& M_{1}=F\left(V_{\max }+V_{1}\right)+F l
\end{aligned}
$$

When there are vertical and axial loads on the arm, it is difficult to calculate the deflection. A simplification is made to help solve the problem: the deflection of scissor arm is made by the axial load. Then the results are obtained:

$V_{1}=\frac{F x\left(l^{2}-x^{2}\right)}{6 E I}$

$V_{2}=\frac{F x^{3}}{6 E I}-\frac{F l x^{2}}{2 E I}-\frac{F l^{2} x}{3 E I}$

$V_{\max }=-\frac{2 F l^{3}}{3 E I}$

when the critical load is assessed, the result is calculated in Matlab and $F_{\text {cr }}=21078 \mathrm{~N}$ is obtained. As for the arm number 2 shown in Fig. (6), there is a hydraulic force at $x=c$, the axial force:

$F_{2}=\left(\frac{W}{2}-P \frac{a \sin \theta}{l \cos \alpha}\right) \sin \alpha=140 \mathrm{kN}$

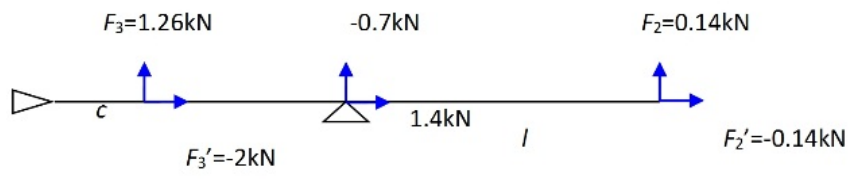

Fig. (6). Boundary condition of the scissor arm number 2 of first case.

Supposing that there are vertical and axial loads on the arm, then for right arm, the deflection is:

$v=\frac{F_{2} x\left(l^{2}-x^{2}\right)}{6 E I}$

For the left arm, it can be divided into two parts: 


$$
\begin{array}{cc}
-\frac{F_{3}(l-c) x\left(x^{2}-l^{2}+(l-c)^{2}\right)}{6 l E I} & 0 \leq x \leq c \\
-\frac{F_{3}(l-x) c\left(x^{2}-l^{2}+(l-c)^{2}\right)}{6 l E I} & c \leq x \leq l
\end{array}
$$

Now the scissor arm is composed of 3 parts

For the first part, $0<x<c$ : the deflection is

$V_{1}=\frac{F_{2} x\left(l^{2}-x^{2}\right)}{6 E I}-\frac{F_{3}(l-c) x\left(x^{2}-l^{2}+(l-c)^{2}\right)}{6 l E I}$

The moment of this part:

$M_{1}=F_{2} l+F\left(V_{\max }+V_{1}\right)+F_{3}(c-x)+F_{3}^{\prime}\left(V_{c}-x\right)$

The deflection at $x=c$ is

$V_{c}=\frac{F_{2} c\left(l^{2}-c^{2}\right)}{6 E I}-\frac{F_{3}(l-c) c\left(c^{2}-l^{2}+(l-c)^{2}\right)}{6 l E I}$

At the second part $c<x<l$, the deflection of this part is

$V_{2}=\frac{F x\left(l^{2}-x^{2}\right)}{6 E I}-\frac{F_{3}(l-x) c\left(x^{2}-l^{2}+(l-c)^{2}\right)}{6 l E I}$

The moment of this part is

$M_{2}=F L+F\left(V_{\max }-V_{2}\right)$

The third part is the right arm:

$V_{3}=-\frac{F x^{3}}{6 E I}+\frac{F L x^{2}}{2 E I}+\left(\frac{F l^{2}}{3 E I}+\frac{F_{3}\left(c^{3}-c l^{2}\right)}{6 l E I}\right) x$

$V_{\max }=\frac{2 F l^{3}}{3 E I}+\frac{F_{3}}{6 E I}\left(c^{3}-c l^{2}\right)$

The moment of this part is

$M_{3}=F\left(V_{\max }-V_{3}+l-x\right)$

Integrating the internal energy $U$ on the three parts of the scissor arm:
$U=\int_{0}^{c}\left(\frac{F^{2} d x}{2 E A}+\frac{F_{3}^{\prime 2} d x}{2 E A}+\frac{M_{1}^{2} d x}{2 E I}\right)$

$+\int_{c}^{l}\left(\frac{F^{2} d x}{2 E A}+\frac{M_{2}^{2} d x}{2 E I}\right)+\int_{0}^{l}\left(\frac{F^{2} d x}{2 E A}+\frac{M_{3}^{2} d x}{2 E I}\right)$

When the critical load is calculated, following is obtained

$\frac{d U}{d F}=0 \quad \frac{d^{2} U}{d F^{2}}>0$

The critical load of the scissor arm number 2 of the first case equals to $=21030 \mathrm{~N}$.

$F_{c r}=\left(\frac{W}{2}-P \frac{a \sin \theta}{l \cos \alpha}\right) \sin \alpha$

$=W\left(\frac{1}{2}-\frac{2 l}{(2 l-c) \sin \theta+c \cos \theta \tan \alpha} \frac{a \sin \theta}{l \cos \alpha}\right) \sin \alpha$

$=21030 \mathrm{~N}$.

The critical axial force and the critical load of the 6 kinds of scissor lifts were calculated by using energy method. All the results are shown in Table 4. Since the fifth case is special, the scissor arm number 1 of the fifth case does not buckle or have a potential of losing stability under the boundary condition that is given. In this paper, the scissor arm number 2 of this case is only studied.

For each case of the scissor lifts, the final critical load equals the smaller critical load of its two scissor arms.

For each case of the scissor lifts, the final critical load equals the smaller critical load of its two scissor arms.

\section{FEM MODELING ANALYSIS OF THE SCISSOR ARM}

Finite element method is an efficient and approximating calculation method. It is also a method of numerical solution for solving field problem. The principle is dividing the continuous solution domain into a finite number of discrete units and finding an approximate solution with the approximate function within each cell hypothesis. Then all the cells are combined to form a corresponding numerical model according to standard methods. In this paper, MSC.Patran and MSC.Nastran were used to analyze the

Table 4. Critical axial force and critical load of the scissor lift.

\begin{tabular}{|c|c|c|c|c|c|}
\hline Case & $\mathbf{1}$ & $\mathbf{2}$ & $\mathbf{3}$ & $\mathbf{4}$ & $\mathbf{6}$ \\
\hline \hline $\begin{array}{c}\text { expression } \\
F_{c r, 2}\end{array}$ & $\frac{W}{2} \sin \alpha$ & $\frac{W-P \sin \theta}{2} \cos \alpha$ & $\frac{W-P \sin \theta}{2} \cos \alpha$ & $\frac{\mathrm{W}}{2} \sin \alpha$ & $\frac{W}{2} \sin \alpha$ \\
\hline $\begin{array}{c}\text { expression } \\
F_{c r, 1}\end{array}$ & $\sin \alpha\left(\frac{1}{2}-P \frac{a \sin \theta}{l \cos \alpha}\right) W$ & $\frac{W}{2} \sin \alpha$ & $\frac{W}{2} \sin \alpha$ & $\left(P \frac{2(l-f) \sin (\theta-\alpha)}{2 l \cos \alpha}-\frac{W}{2}\right) \cos \alpha$ & $\left(\frac{W}{2}-P \frac{a \sin \theta}{l \cos \alpha}\right) \cos \alpha$ \\
\hline$F_{c r, 1}$ & 21078 & 21080 & 5240 & 16300 & $\frac{W}{2} \cos \alpha$ \\
\hline$F_{c r, 2}$ & 21030 & 20890 & 21080 & 21080 & 18700 \\
\hline$W_{c r, 1}$ & 59600 & 33460 & 52400 & 46110 & 21080 \\
\hline$W_{c r, 2}$ & 297300 & 59090 & 59630 & 58560 & 62900 \\
\hline
\end{tabular}


static stability of the scissor lift and the critical load of different cases were analyzed in Nastran by using buckling analysis.

Here, the first case is taken as an example. The main steps of modeling in Patran are as follows:

1. Building the geometry model: a curve is built, with its length as $1200 \mathrm{~mm}$, from the point $(0,0,0)$ to the point $(1200,0,0)$.

2. Building meshes: one dimension element is built to the curve and 101 nodes are provided.

3. Defining material: aluminum is provided to the scissor arm: Young's modulus equals $70 \mathrm{GPa}$ and poison ratio equals 0.3 .

4. Defining property: beam property (1D) is given to the arms.

5. Defining boundary conditions: boundary conditions correspond to the model's hypotheses

6. Defining load: the load corresponds to the model's hypotheses, and a force of $\langle-707,-707,0\rangle$ is added to the node of the right edge.

7. Analysis: buckling analysis is chosen and the buckling factor of first degree is obtained.

8. Accessing results: the result of Nastran and the buckling factor in patran are obtained.
The two scissor arms of the second case of scissor lift are taken as examples which are modeled as type in Fig. (7). The buckling results are shown in Figs. $(\mathbf{8}, \mathbf{9})$.

The same analysis and the corresponding results of single scissor arm buckling critical load factor can be obtained through each of the six kinds of scissor lifts. All the buckling factors and critical loads of the six kinds of scissor lifts are shown in Table 5. For each case, the smaller critical load of the two scissor arms is the critical load of the scissor lift.

Similar to the results of the energy method, the scissor arm number 1 of the fifth case does not have a stability problem in the hypothesis of boundary condition as given in the paper. Only the stability of scissor arm number 2 of fifth case is considered. The results of energy method and modeling method of the six cases of scissor lifts were taken and a comparison was made. The result is shown in Table $\mathbf{6}$.

\section{STATIC STABILITY ANALYSIS OF SCISSOR LIFT MODEL}

The scissor lift model studied in this factor included two scissor arms and a hydraulic actuator which was a cylinder model with the material of steel. Static stability analysis of the model included scissor arm instability and hydraulic cylinder instability.

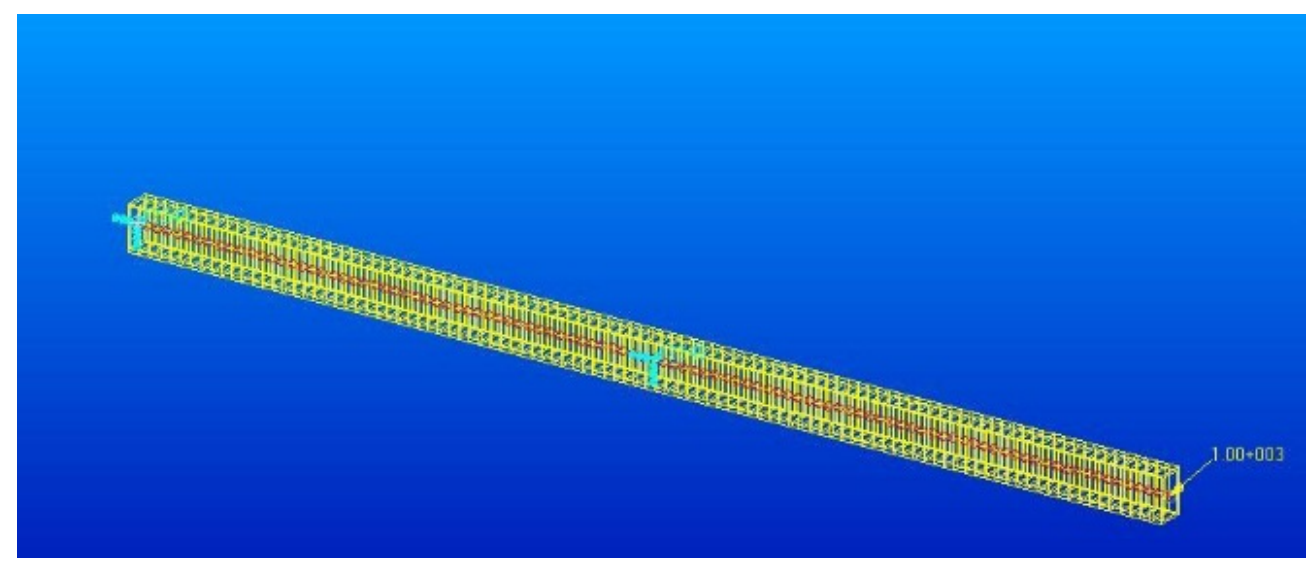

Fig. (7). The modeling of scissor arm in Patran.

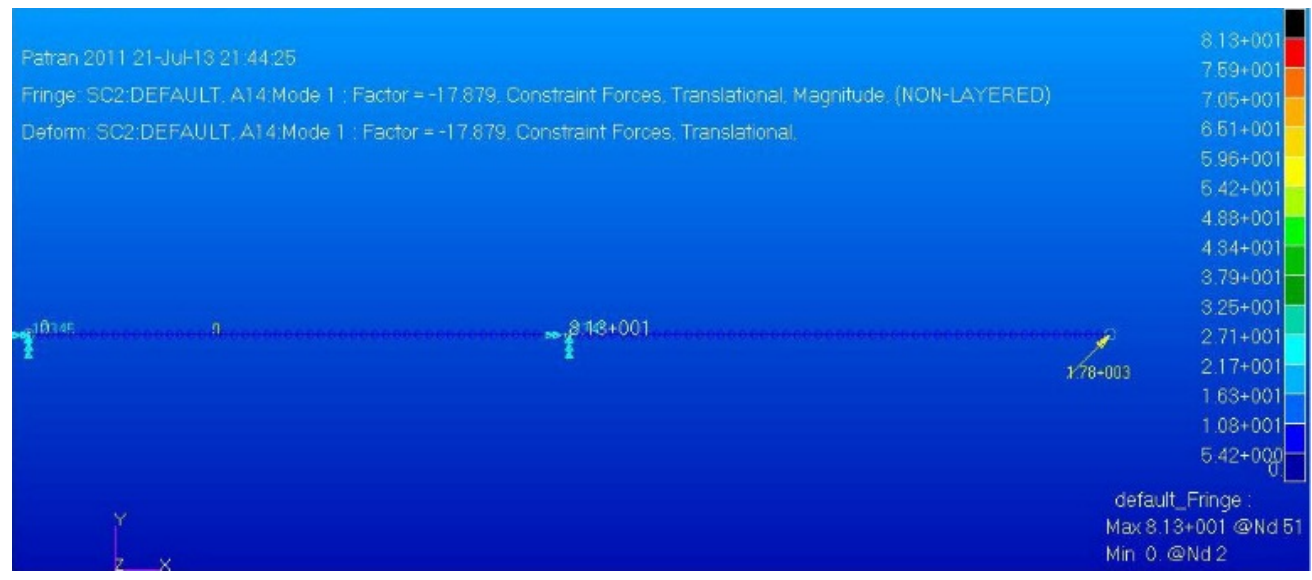

Fig. (8). Buckling results of the scissor arm number 1 of second case. 


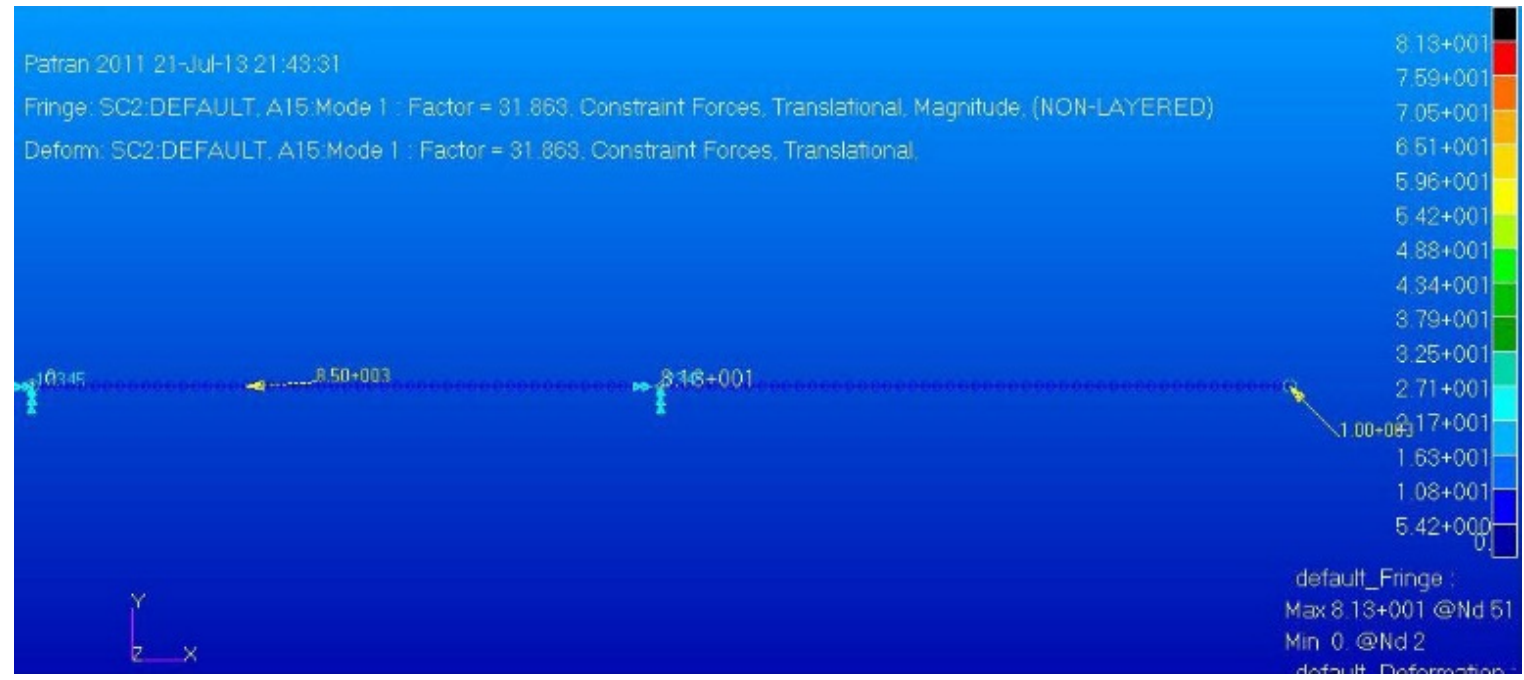

Fig. (9). Buckling results of the scissor arm number 2 of second case.

Table 5. Buckling factors and critical load of six cases of scissor lifts.

\begin{tabular}{|c|c|c|c|c|c|c|}
\hline Case & $\mathbf{1}$ & $\mathbf{2}$ & $\mathbf{3}$ & $\mathbf{4}$ & $\mathbf{5}$ & $\mathbf{6}$ \\
\hline \hline W_energy (N) & 59600 & 33460 & 52400 & 46110 & 112700 & 62900 \\
\hline W_modeling (N) & 63730 & 35760 & 52800 & 46930 & 120500 & 64190 \\
\hline Difference (\%) & 6.5 & 6.4 & 0.76 & 1.7 & 6.5 & 2.0 \\
\hline
\end{tabular}

Table 6. Comparison of energy method and modeling method of scissor arms' static stability.

\begin{tabular}{|c|c|c|c|c|c|c|}
\hline Case & $\mathbf{1}$ & $\mathbf{2}$ & $\mathbf{3}$ & $\mathbf{4}$ & $\mathbf{5}$ & $\mathbf{6}$ \\
\hline \hline Factor1 & 31.86 & -17.879 & -26.413 & -23.462 & 32.093 \\
\hline Factor2 & 160.91 & 31.86 & 31.863 & -31.228 & 60.23 & 31.863 \\
\hline$F_{c r, 1}(\mathrm{~N})$ & 22530 & 22530 & 5280 & 16590 & 22690 \\
\hline$F_{c r, 2}(\mathrm{~N})$ & 22530 & 22530 & 22530 & 22480 & 22530 & 22530 \\
\hline$W_{c r, 1}(\mathrm{~N})$ & 63730 & 35760 & 52800 & 46930 & 64190 \\
\hline$W_{c r, 1}(\mathrm{~N})$ & 318670 & 63730 & 63730 & 62460 & 120500 & 63730 \\
\hline
\end{tabular}

The property of the hydraulic actuator was one dimension element with Young's Modulus equaling to 200 Gpa and Poisson ratio equaling 0.3 .

When the model of scissor lift was made, the lifting angles equaling to 15 degrees, 30 degrees and 45 degrees were selected. The first case with lifting angle equaling to 45 degree was taken as an example; the model is shown in Fig. (10).

For any kind of scissor lift, the minimum critical load value in the three cases is the critical load of the case. The critical buckling load factor and the modeling results obtained are shown in Table 7.

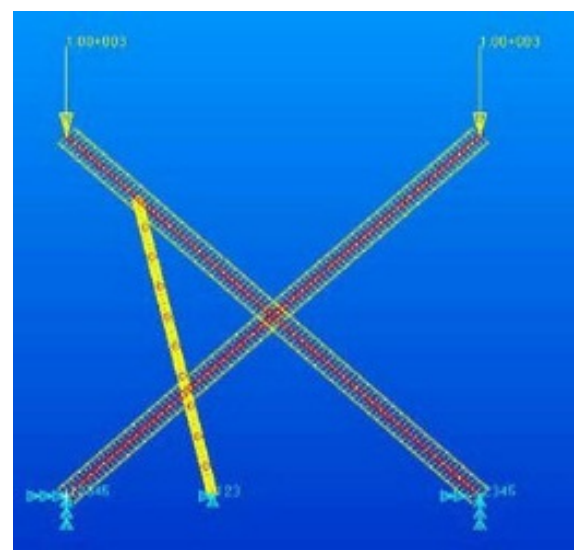

Fig. (10). Model of the first case of scissor lift. 
Table 7. The buckling factor and critical load of scissor lift models.

\begin{tabular}{|c|c|c|c|c|c|c|}
\hline Case & $\mathbf{1}$ & $\mathbf{2}$ & $\mathbf{3}$ & $\mathbf{4}$ & $\mathbf{5}$ & $\mathbf{6}$ \\
\hline \hline Factor_15 & 12.56 & 1.97 & 1.43 & 13.59 & 35.755 & 11.88 \\
\hline W_15(N) & 25120 & 3940 & 2860 & 27180 & 71510 & 23760 \\
\hline Factor_30 & 11.25 & 2.65 & 3.07 & 11.64 & 67.803 & 10.03 \\
\hline W_30(N) & 22500 & 5300 & 6140 & 23280 & 135606 & 20060 \\
\hline Factor_45 & 9.89 & 3.22 & 5.2 & 10.01 & 87.939 & 8.70 \\
\hline W_45(N) & 19780 & 6440 & 10400 & 20020 & 175878 & 17400 \\
\hline Critical load (N) & 19780 & 3940 & 2860 & 20020 & 71510 & 17400 \\
\hline
\end{tabular}

After modeling and calculating critical loads of six kinds of scissor lifts, the order of static stability of six cases from good to bad is: $5,4,1,6,2,3$.

\section{CONCLUSION}

This paper studied the static stability of six kinds of scissor lifts with one input force of hydraulic actuator by having the input on the lines of the nodes of the scissor lifts. The static stability of six kinds of scissor lifts was compared.

The results of the stability analysis of single scissor arm obtained by two methods are basically consistent which verify the accuracy of the single scissor arm critical load. When the finite element model was used to analyze the stability, the method Eigen value buckling analysis was used. The results of modeling and simulation were slightly greater than the energy analysis results because the Eigen value buckling analysis result was considered as the upper limit of the critical load.

The stability results of single scissor arm and the scissor lift were compared. The results obtained from single scissor arm were better than the overall scissor lift model which may be due to its stronger boundary conditions. The results of the overall model were closer to the actual situation. The analysis of the single arm model, made it easier to compare the theoretical solutions and modeling solutions thus the study of stability of single scissor arm has proved to be meaningful.

\section{CONFLICT OF INTEREST}

The authors confirm that this article content has no conflict of interest.

\section{ACKNOWLEDGEMENTS}

This work is supported by National Natural Science Foundation of China and Civil Aviation Administration of China jointly funded project (Grant \# U1233106), the Fundamental Research Funds for the Central Universities funded project (Grant \# ZXH2012H007) and the university scientific research project of Civil Aviation University of China (Grant \# 2012KYE05). Corresponding author is grateful to all who provided helps for this research.

\section{REFERENCES}

[1] E.L. Newlin, Work Platform Lift Machine with Scissor Lift Mechanism Employing Telescopable Electro-Mechanism Lift Actuation Arrangement, US Patent US6044927A, 2000.

[2] T. Liu, and J. Sun, "Simulative calculation and optimal design of scissor lifting mechanism," In: Chinese Control and Decision Conference, Guilin, 2009, pp. 2079-2082.

[3] H. Tian, and Z. Zhang, "Design and simulation based on pro/E for A hydraulic lift platform in scissors type," Journal of SciVerse Science Direct, vol.16, pp.772-781, 2011.

[4] A. Yenal, and G. C. Sobek, "Werner A novel adaptive spatial scissor-hinge structural mechanism for convertible roofs", Journal of Engineering Structures, vol. 33, no. 4, pp. 1365-1376, 2011.

[5] K. Guo, C. Pan, and X. Zhang, "A method of establishing and analyzing universal kinematics model based on plane scissor-like element, " Journal of Machine Design and Research, vol. 26, no. 6, pp. 27-30, 2010.

[6] I. Raskin, "Stiffness and Stability of Deployable Pantographic Columns", PhD Thesis, University of Waterloo, Waterloo, Ontario, 1998. 